Artículo de Investigación

Apuntes del CENES

ISSN 0120-3053

Volumen $33-\mathrm{N}^{\circ} .57$

Enero - Junio de 2014

Págs. 11-30

\title{
Series de días hábiles en Colombia: una aplicación al ajuste del crecimiento industrial
}

\author{
Working days series in Colombia: an \\ application to industrial growth adjustment
}

\author{
Álvaro Montenegro García*
}

Fecha de recepción: 14 de junio de 2013

Concepto de evaluación: 14 de febrero de 2014

Fecha de aprobación: 21 de marzo de 2014

Deseo agradecer a Nicolás Ronderos Pulido, Alfredo Contreras Eitner y a Santiago de la Cuesta Ávila por su valiosa ayuda en la elaboración de este trabajo. Las opiniones expresadas son del autor y no comprometen a la institución.

PhD en Economía, New York University. Ingeniero Electrónico, Universidad Javeriana. Profesor Titular del Departamento de Economía, Universidad Javeriana. Bogotá. Colombia. Correo electrónico: amontene@javeriana.edu.co 


\section{Resumen}

Si bien muchos economistas son conscientes del efecto de los días hábiles sobre la medición de la producción, aún es frecuente encontrar que estas cifras se analizan sin el correspondiente ajuste. En general, el número de días hábiles en un mes cambia de un año a otro, lo cual es cierto para todos los meses, no solo para los que contienen la Semana Santa. Este trabajo recopila la serie de días hábiles mensuales tomando en cuenta los festivos actuales y anteriores a la Ley Emiliani, y la utiliza para ajustar la serie de producción manufacturera del DANE. Para algunos meses, la diferencia entre la cifra de crecimiento industrial reportada por el DANE y la cifra ajustada por días hábiles puede acercarse a los 12 puntos porcentuales, en cualquier dirección.

Palabras clave: producción industrial, encuesta mensual manufacturera, DANE, días hábiles, estacionalidad.

JEL: E23, C82

\section{Abstract}

While many economists are aware of the effect of workdays on production measures, it is common to find data being analyzed without adjustment. Generally, the number of workdays in a month changes from one year to the next. This is true for all months, not only for March and April which exhibit the largest variations due to the Holy Week. This paper constructs a series of workdays, accounting for changes in holidays through time, and uses it to adjust industrial production data from DANE. Some months the difference between industrial growth reported by DANE and growth adjusted by workdays may reach 12 percentage points, positive or negative.

Keywords: industrial production, monthly manufacturing survey, working days, seasonality. 


\section{INTRODUCCIÓN}

Algunas variables económicas son sensibles al número de días hábiles en el mes; una de ellas es la producción manufacturera. Puede esperarse que los meses con mayor número de días hábiles arrojen mayor producción que los meses con menor número de días hábiles. Esto implica que si el número de días hábiles para un mes cambia de un año a otro, las comparaciones de un mes contra el mismo mes del año anterior pueden dar una idea equivocada del crecimiento en la producción. Esta consideración cobra mayor importancia al recordar que una fuente principal de información para la estimación del producto interno bruto (PIB) son las cifras de producción manufacturera. También porque dichas cifras se utilizan para ejercicios de predicción del crecimiento en el PIB trimestral, en razón de que se producen mensualmente y con menor rezago en comparación con el más lento proceso de recolección de otras cifras que componen el PIB.

En la experiencia internacional, este problema de la comparación intertemporal de las series de producción lo abordan las oficinas de estadística ajustando por días hábiles o realizando un ajuste estacional [ver, por ejemplo, Eurostat (2006, subcapítulo 5.6), Statistics Canada (2014), Federal Reserve (2003, p. 161)]. El ajuste por días hábiles toma en cuenta las particularidades de cada mes de manera individual mientras que el ajuste estacional toma en cuenta el promedio de las particularidades de cada mes. En los casos en los cuales se hacen ambas transformaciones, usualmente, el ajuste estacional se aplica sobre la serie ajustada por días hábiles. Diversos métodos para el modelaje y manejo del 
número de días hábiles pueden consultarse en Soukup y Findley (2000) o Landiray (2006).

Se trata de ajustar la serie de producción manufacturera de manera que refleje la producción por día hábil en lugar de la producción total del mes. Así será posible determinar qué parte del cambio en la cifra mensual se debe a un cambio en el número de días hábiles y qué parte corresponde a un aumento real. Los algoritmos de desestacionalización no bastan para solucionar este problema, ya que se ocupan del comportamiento promedio y no incluyen calendarios adecuados que reflejen el número de días hábiles para cada mes de manera individual.

Para efectuar el ajuste descrito es necesario dividir la serie de producción mensual por la serie de días hábiles mensuales y luego realizar las comparaciones con la serie resultante. Aunque es un problema largamente conocido, en Colombia pocas discusiones involucran el ajuste por días hábiles de manera sistemática [para algunas excepciones, ver Martínez (2004), Banco de la República (2005) y Montenegro (2010)].

En este trabajo se utiliza la serie de producción real de la Muestra mensual manufacturera del DANE (sin trilla de café) desde enero de 1980 hasta enero 2013. Dicha serie es un índice con base 2001. Por otro lado, la serie de días hábiles se presenta para el período 1980 2021, extendiéndose más allá del 2013, con el objeto de que pueda ser utilizada por los investigadores y analistas en los años venideros para ajustar las cifras de producción a medida que vayan siendo producidas por el DANE. Adicionalmente, si el investigador lo requiere, esta serie podrá ser agregada para obtener, por ejemplo, una serie de días hábiles trimestral o anual.

\section{LA SERIE DE DÍAS HÁBILES}

Para el conteo del número de días hábiles por mes se utilizó el calendario encontrado en la dirección http:// www.cuandoenelmundo.com/calendario/ Consultado para Colombia, el algoritmo de este calendario aplica a todos los años la normatividad más reciente sobre días de fiesta. Obviamente, debe tenerse en cuenta que las fiestas han cambiado a lo largo del tiempo. Por ejemplo, solicitando el calendario para el año 1800 , se encuentran días de fiesta que no existían en esa época, como mayo 1 (Día del Trabajo), 20 de julio (Día de la Independencia), etc. El mencionado calendario es útil solo después de 1983, puesto que la normatividad actual se deriva de la Ley 51 del 22 de diciembre de 1983, conocida como Ley Emiliani. Esta ley trasladó al lunes varios de los festivos: "el descanso remunerado del seis de enero, diecinueve de marzo, veintinueve de junio, quince de agosto, doce de octubre, primero de noviembre, 
once de noviembre, Ascensión del Señor, Corpus Cristi y Sagrado Corazón de Jesús cuando no caigan en día lunes se trasladarán al lunes siguiente a dicho día".

Para los años anteriores a la entrada en vigencia de la Ley Emiliani, esto es, para 1980, 1981, 1982 y 1983, se utilizaron calendarios físicos de la época. Adicionalmente, se utilizaron calendarios físicos para otros años, realizando un conteo independiente a manera de comprobación.

Se construyeron dos series de días hábiles para el período enero de $1980 \mathrm{a}$ diciembre de 2021: con sábados (Anexo) y sin sábados (disponible a solicitud). En lo que sigue se utilizará la serie que incluye sábados (que cuenta los días del mes menos domingos y festivos) como días hábiles, ya que buena parte de la industria labora sábados.

La serie con sábados, se muestra en la Figura 1. Puede observarse que el rango del número de días hábiles por mes oscila entre 22 y 27 , lo cual sugiere un potencial significativo de variabilidad. La Figura 2 muestra el histograma y algunas estadísticas descriptivas de la serie. $\mathrm{Su}$ media indica que el mes típico tiene 24.7 días hábiles contando sábados. La desviación estándar es cercana a la unidad. Del histograma se observa que la mayoría de los meses tienen 24 y 25 días y que, en proporción decreciente, siguen meses con $26,23,27$ y 22 días.

La variación en días hábiles se observa mejor al tomar cambios porcentuales de un mes contra el mismo mes del año anterior. Esta serie se muestra en la Figura 3 y sus cifras en el Anexo. La Figura 4 muestra el histograma y algunas estadísticas descriptivas. La variación porcentual en el número de días hábiles de un año a otro para el mismo mes oscila entre menos $11.5 \%$ y más $13 \%$; en otras palabras, hay meses que tienen $11.5 \%$ menos días hábiles o $13 \%$ más días hábiles en comparación con el mismo mes del año anterior. El impacto sobre la producción real es evidente. Aproximadamente 230 de los 492 meses de la muestra tienen cero cambio porcentual y 180 tienen $4 \%$ o $-4 \%$, lo cual equivale más o menos a un día. Queda un número sustancial de meses con variaciones de 2 o 3 días. 
Series de días hábiles en Colombia: una aplicación al ajuste del crecimiento industrial Álvaro Montenegro García

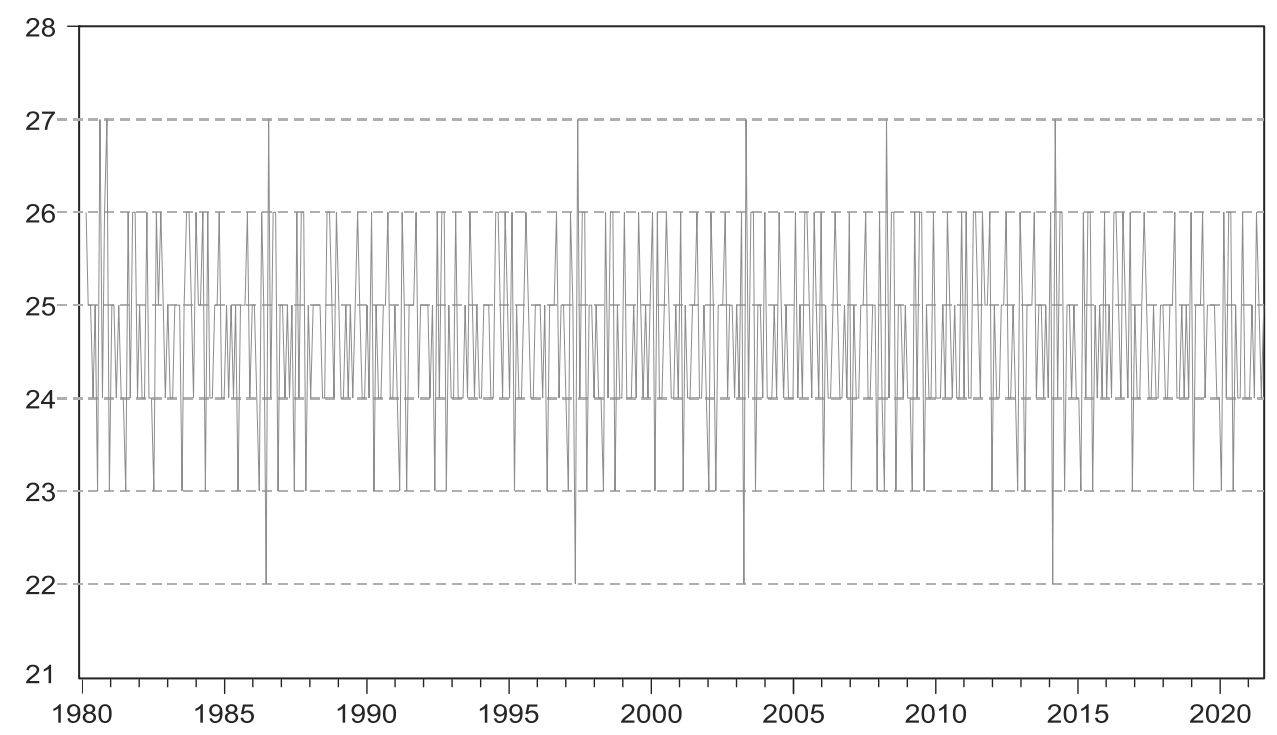

Figura 1. Días hábiles mensuales.

Fuente: elaboración del autor.

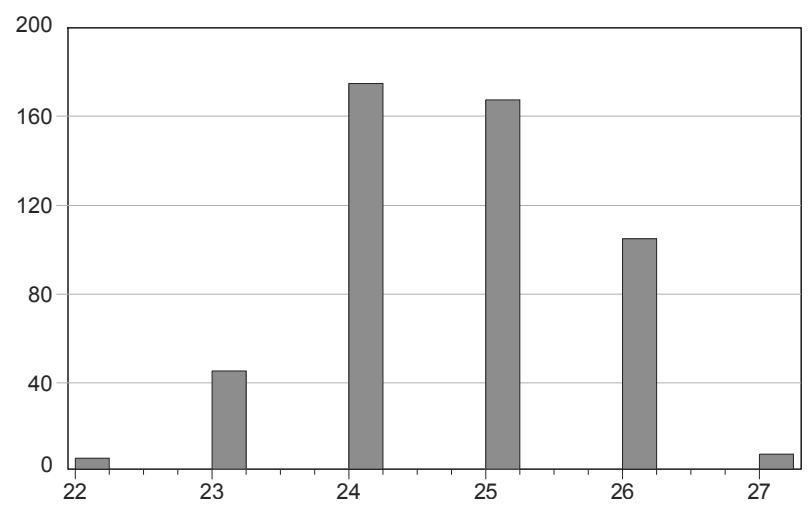

Series: DH

Sample 1980M012021M12

Observations 504

$\quad 24.68849$

Median $\quad 25.00000$

Maximum $\quad 27.00000$

Minimum 22.00000

Std. Dev. $\quad 0.968791$

Skewness $\quad-0.040671$

Kurtosis $\quad 2.528764$

Jarque-Bera $\quad 4.802270$

\begin{tabular}{ll} 
Probability & 0.090615 \\
\hline
\end{tabular}

Figura 2. Días hábiles mensuales

Fuente: elaboración del autor. 


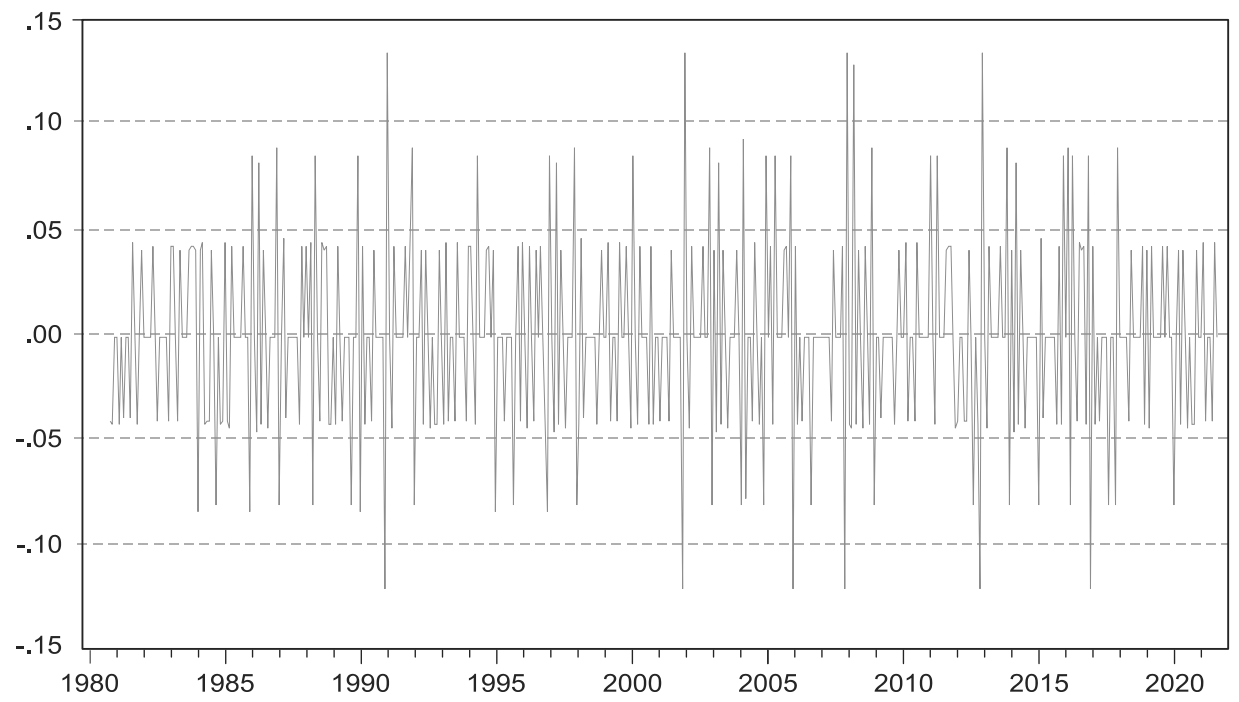

Figura 3. Días hábiles mensuales. Cambio porcentual con el mismo mes del año anterior. Fuente: elaboración del autor.
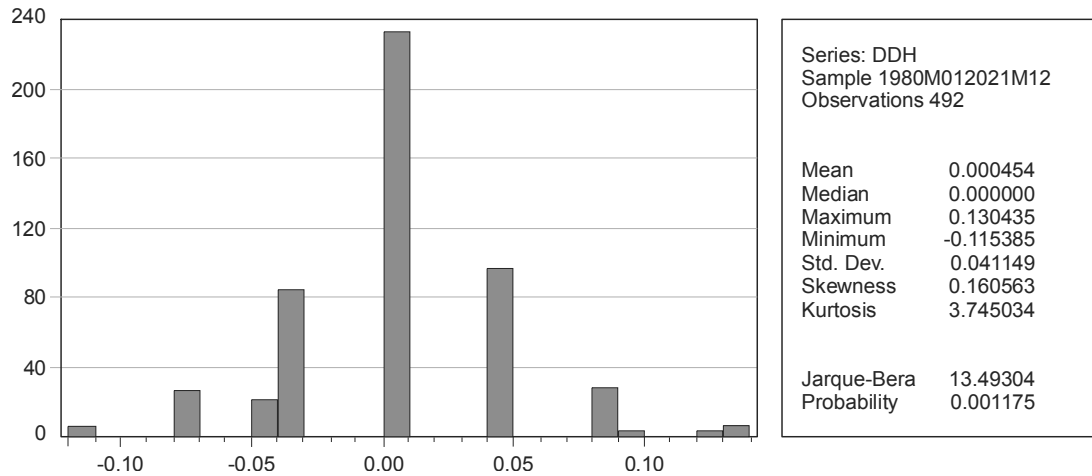

Figura 4. Días hábiles mensuales. Cambio porcentual con el mismo mes del año anterior. Fuente: elaboración del autor.

La explicación de un par de casos puntuales del Anexo puede ser ilustrativa. Por ejemplo, el número de días hábiles de abril 2013 respecto a abril 2012 creció
$13.04 \%$, explicado porque abril 2013 tuvo 26 días hábiles contando sábados, mientras que abril 2012 tuvo 23. De esta diferencia, dos días se explican por el hecho de que 
la Semana Santa de 2013 se celebró en el mes de marzo mientras que en el 2012 se celebró en el mes de abril. El día hábil adicional se explica porque abril 2012 tuvo 5 domingos mientras que abril de 2013 tuvo 4.

El número de días hábiles de julio de 2008 respecto a julio de 2007 creció $12.5 \%$, explicado porque julio de 2008 tuvo 27 días hábiles contando sábados, mientras que julio de 2007 tuvo 24. Uno de esos tres festivos de diferencia está explicado por la celebración del feriado de San Pedro y San Pablo. Dicha festividad, que se celebra el 29 de junio, cayó en domingo para el año 2008 y se trasladó al siguiente lunes por la Ley Emiliani; el siguiente lunes fue 30 de junio, por lo que el festivo no perteneció al mes de julio. Caso contrario ocurrió en el 2007, cuando el 29 de junio fue un viernes y el siguiente lunes fue el 2 de julio. De esta forma, el festivo no alcanzó a caer en el mes de junio y se trasladó al mes de julio. Otro festivo de diferencia está explicado por la celebración del Día de la Independencia. Dicha festividad se celebra el 20 de julio y no está cobijada por la Ley Emiliani, lo que implica su carácter inamovible. En el 2008, el 20 de julio fue domingo y por ende no generó ningún cambio en cuanto al número de días festivos. En contraste, en el 2007, el 20 de julio fue un viernes, lo que agregó un festivo más al mes. El festivo de diferencia restante corresponde a que julio de 2008 tuvo 4 domingos y julio de 2007 tuvo 5 domingos, ya que el primero empezó un martes (y por ende no alcanzó un quinto domingo), mientras que el último empezó un domingo, debido a que el año 2008 fue bisiesto.

\section{SEMANA SANTA}

Un factor importante para explicar la diferencia en el número de días hábiles es la fecha de la Semana Santa, aunque no es el único factor. Dicha fecha introduce las mayores variaciones, no solo de mes a mes sino también de trimestre a trimestre, porque suele alternar entre marzo (primer trimestre) y abril (segundo trimestre), aunque ocasionalmente cae en el mismo mes (mismo trimestre) dos años seguidos, como sucedió en 2006 y 2007, cuando cayó en abril. La fecha de la Resurrección del Señor, que marca el final de la Semana Santa y que ocurre entre el 22 de marzo y el 25 de abril, corresponde al siguiente domingo después del primer plenilunio que se presente en o a partir del equinoccio primaveral. Si la primera luna llena cae domingo, entonces se celebra al domingo siguiente. Los equinoccios ocurren dos veces al año, en primavera y otoño, cuando el eje vertical de la tierra es ortogonal a una línea trazada desde el sol hasta el centro de la tierra; esto es, cuando ambos hemisferios están a la misma distancia del sol. El de primavera ocurre alrededor de marzo 20 o 21. Sin embargo, para efectos prácticos, se tomó el 21 de marzo como fecha del equinoccio. La luna llena puede presentarse entre la fecha del equinoccio y casi un mes después (lo que tarda el ciclo lunar), luego de lo cual hay que esperar al domingo siguiente, lo cual explica la variabilidad en la fecha de Semana Santa. 


\section{ESTACIONALIDAD}

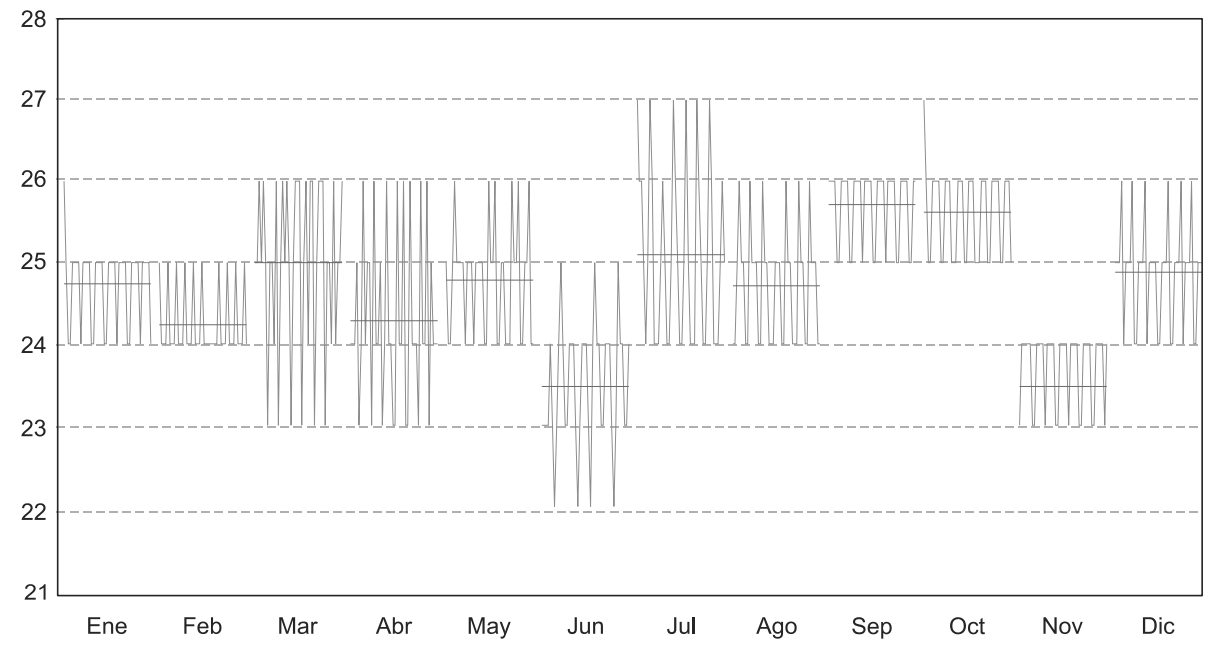

Figura 5. Estacionalidad de los días hábiles mensuales Fuente: elaboración del autor.

La Figura 5 muestra el comportamiento estacional (en número de días) de la serie de días hábiles mensuales. Las observaciones se agrupan por mes y oscilan alrededor del promedio del mes (denotado por una línea horizontal en cada mes). Junio es el mes con el promedio más bajo de días hábiles, seguido por noviembre. En contraste, los promedios más altos son los de septiembre y octubre. Los meses con mayor volatilidad son marzo, abril y julio. Nótese que ningún mes tiene el mismo número de días hábiles año tras año. Para una explicación del ajuste estacional ver, por ejemplo, Montenegro (2011).

La Figura 6 muestra el comportamiento estacional para la serie de cambios porcentuales anuales donde es evidente la poca estabilidad en el número de días mensuales, en especial para marzo, abril, julio, agosto y diciembre. Esto sugiere que dividir la producción manufacturera por el número de días hábiles puede tener un efecto significativo en esos meses. 
Series de días hábiles en Colombia: una aplicación al ajuste del crecimiento industrial Álvaro Montenegro García

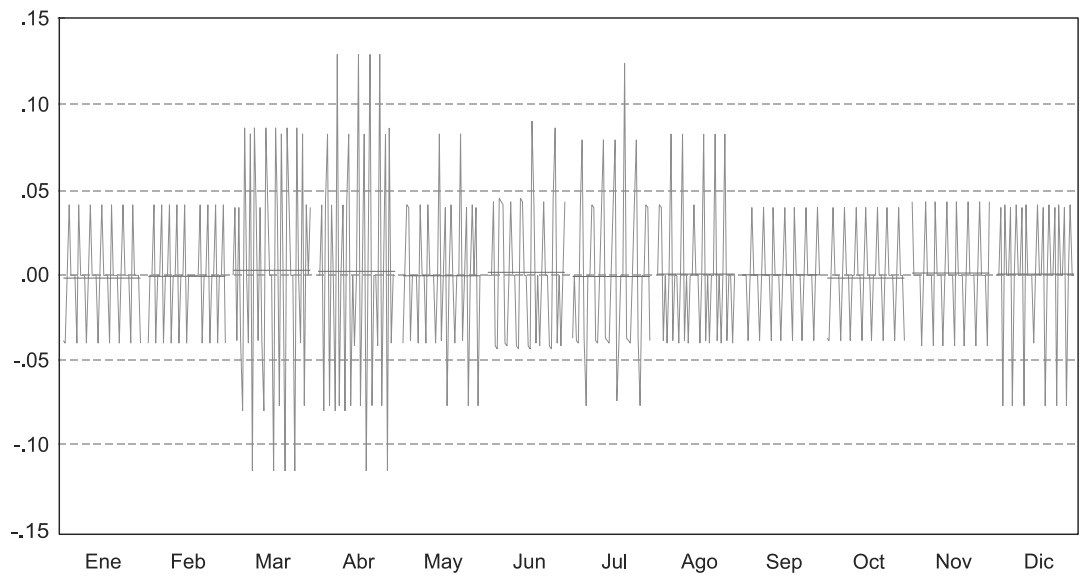

Figura 6. Estacionalidad de los días hábiles mensuales. Cambio porcentual contra el mismo mes del año anterior. Fuente: elaboración del autor.

Al igual que sucede en el orden mensual, ningún trimestre mantiene un número constante de días hábiles año tras año. El número de días hábiles puede variar hasta en $7 \%$ en comparación con el mismo trimestre del año anterior; los datos trimestrales se muestran en la Figura 7.
Incluso los años completos tampoco tienen un número constante de días hábiles, llegando a variar hasta en $1 \%$; los datos anuales se muestran en la Figura 8. En las gráficas se aprecia el efecto de la entrada en vigencia de la Ley Emiliani a partir de 1984.

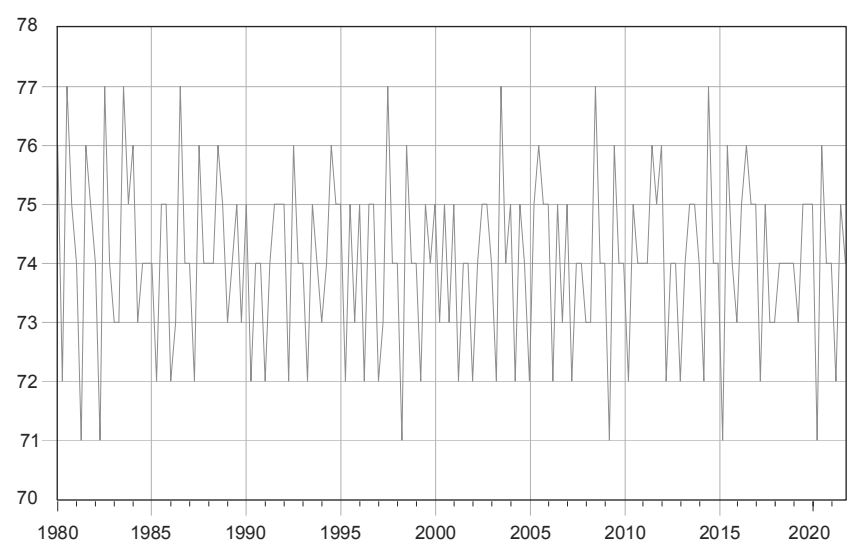

Figura 7. Días hábiles trimestrales Fuente: elaboración del autor. 


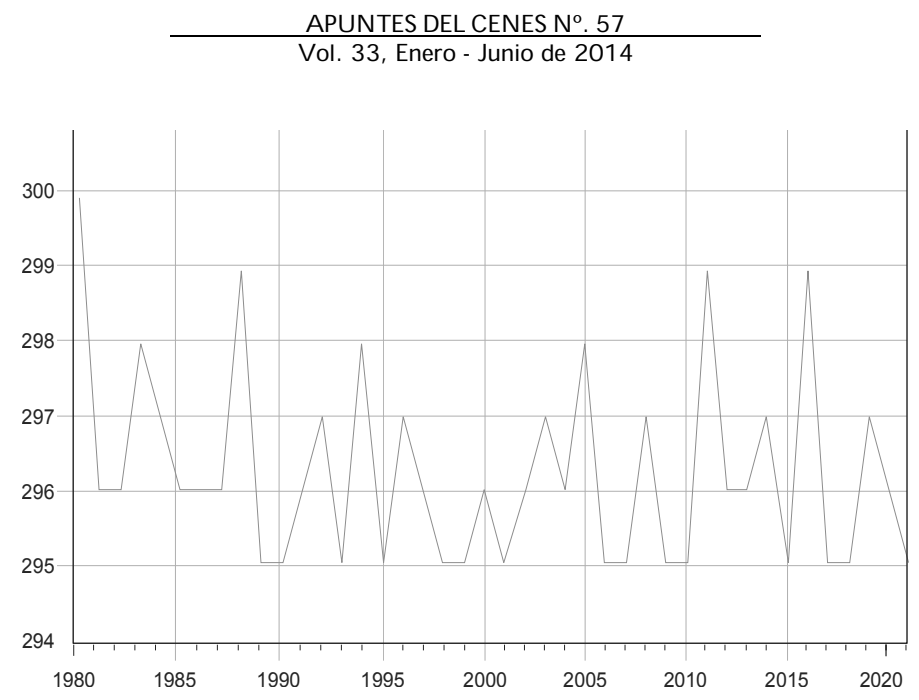

Figura 8. Días hábiles anuales

Fuente: elaboración del autor.

\section{PRODUCCIÓN REAL}

Las cifras de producción mensual real (sin trilla de café) son de la Muestra mensual manufacturera del DANE para el período enero de 1980 a enero de 2013 y corresponde a un número índice con año base 2001. La Figura 9 muestra la gráfica de este índice. La encuesta recopila series adicionales, no estudiadas aquí, pero que igualmente deben ser ajustadas por la serie de días hábiles, como son el empleo permanente y temporal, y las ventas. También mide la variable horas hombre trabajadas en el área de producción (excluye horas trabajadas en el área administrativa).

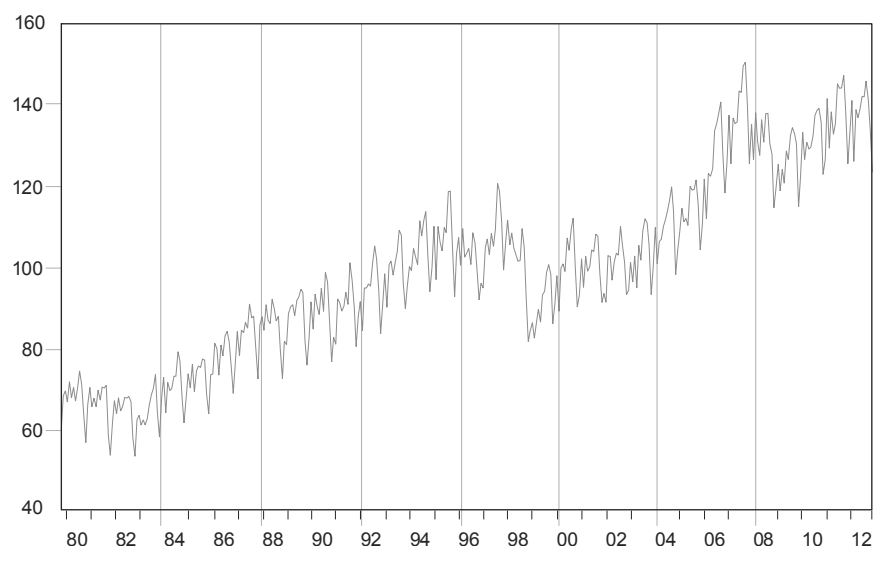

Figura 9. Producción real mensual

Fuente: elaboración del autor con base en datos de la MMM del DANE.

Fuente: elaboración del autor. 
La variable horas trabajadas muestra alguna correlación con la variable días hábiles $\mathrm{y}$, en principio, podría ser utilizada para ajustar la serie de producción en lugar de ajustar por días hábiles. Sin embargo, la cantidad de horas trabajadas depende tanto del número de días hábiles en el mes como de la intención del empresario de incrementar o disminuir su producción. Al usar la cantidad de horas trabajadas para ajustar la serie de producción, estaríamos cancelando parte de este último efecto, que es el que queremos medir en la serie de producción real. En otras palabras, cuanto mayor sea el componente de días hábiles dentro de la serie de horas trabajadas, más útil será para ajustar, pero cuanto mayor sea el componente de intención de aumentar la producción por parte del empresario, menos útil será para ajustar.

Las Figuras 10 y 11 muestran cambios porcentuales anuales, esto es, cambios porcentuales con respecto al mismo mes del año anterior. El cambio en la Figura 10 es el reportado por el DANE como crecimiento mensual de la producción. El cambio mostrado en la Figura 11 es el crecimiento de la serie de producción real dividida por la serie de días hábiles; en otras palabras, es el cambio porcentual en la producción mensual por día hábil. Es la diferencia en estas dos series la que interesa contrastar.

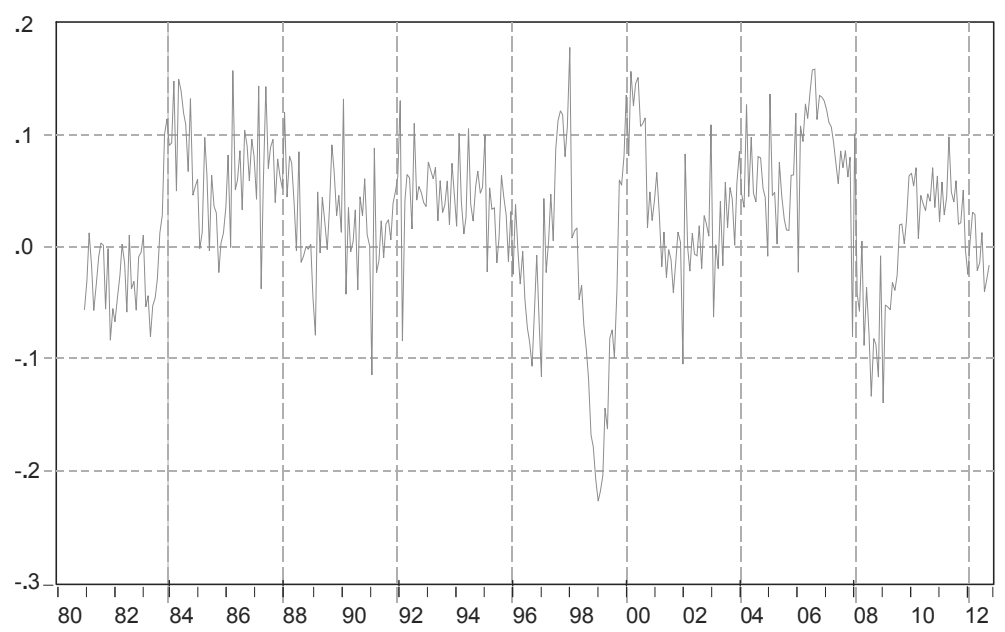

Figura 10. Producción real mensual.

Cambio porcentual con el mismo mes del año anterior.

Fuente: elaboración del autor con base en datos de la MMM del DANE. 


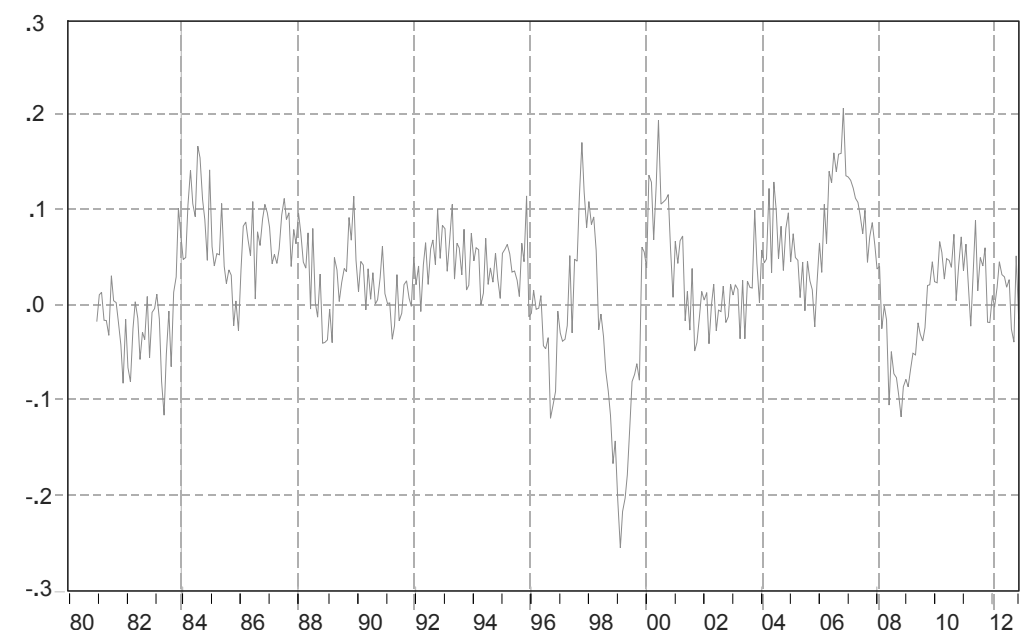

Figura 11. Producción real mensual, promedio por día hábil.

Cambio porcentual con el mismo mes del año anterior.

Fuente: elaboración del autor con base en datos de la MMM del DANE.

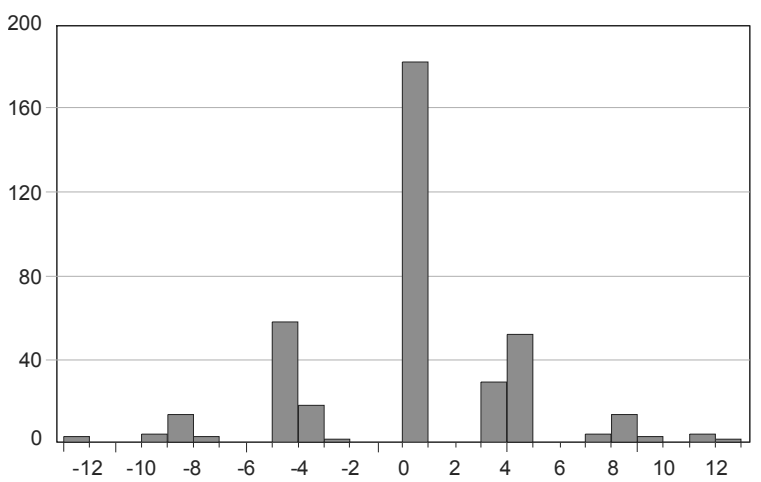

Series: Diferencia con DANE Sample 1980M012013M01 Observations 385

Mean $\quad 0.025691$

Median $\quad 0.000000$

Maximum $\quad 12.74325$

Minimum $\quad-12.69967$

Std. Dev. $\quad 4.158800$

Skewness $\quad-0.076281$

Kurtosis $\quad 3.693295$

Jarque-Bera 8.083938

$\begin{array}{ll}\text { Probability } & 0.017563\end{array}$

Figura 12. Cifras ajustadas menos cifras del DANE.

Diferencia en puntos porcentuales.

Fuente: elaboración del autor con base en datos de la MMM del DANE. 
La Figura 12 muestra el histograma, junto con algunas estadísticas descriptivas, de la diferencia en las dos series de crecimiento anteriores. Específicamente, se trata de la serie del crecimiento en la producción ajustada por días hábiles, menos el crecimiento en la serie de producción que reporta el DANE. El rango de variación es de más de 12 puntos porcentuales hacia arriba o hacia abajo, una cifra significativa considerando que se trata de producción real y no nominal. En otras palabras, para algunos meses la diferencia entre la cifra reportada por el DANE y la cifra ajustada por días hábiles, más cercana a la realidad, puede acercarse a los 12 puntos porcentuales en cualquier dirección.

Para un mes particular se puede corregir la cifra de crecimiento industrial real publicada por el DANE utilizando la fórmula,

$$
\left[\frac{1+\text { cambio encuesta }}{1+\text { cambio días }}-1\right] \times 100
$$

donde cambio de encuesta es el cambio porcentual reportado por el DANE (en decimales) con respecto al mismo mes del año anterior y cambio días es el cambio porcentual (en decimales) en el número de días hábiles con respecto al mismo mes del año anterior (Anexo). Por ejemplo, para diciembre de 2012 las cifras del DANE muestran una caída en la producción real cercana al $3 \%$ mientras que las cifras de días hábiles (Anexo) muestran una caída de $7.69 \%$, lo que arroja

$$
\left[\frac{1+(-0.03)}{1+(-0.0769)}-1\right] \times 100=5.08
$$

por ciento como el crecimiento en la producción real industrial; positivo $5.08 \%$ en lugar del negativo $3 \%$ que había reportado el DANE.

De igual manera se pueden realizar los ajustes para otros meses. Por ejemplo, en marzo de 2013, la Encuesta registró una caída de $11.7 \%$ en la producción, mientras que la cifra ajustada por días hábiles permanecía estable, debido a que marzo 2013 tuvo $11.5 \%$ menos días que marzo de 2012; en abril de 2013, la Encuesta registró un aumento de $8.2 \%$, mientras que la cifra ajustada por días hábiles cayó $4.2 \%$, porque abril de 2013 tuvo $13 \%$ más días hábiles que abril de 2012; en diciembre de 2012, la Encuesta registró una disminución en la producción de $3.05 \%$, mientras que la cifra ajustada por días hábiles subía $5 \%$.

\section{CONCLUSIÓN}

Las cifras de la Encuesta mensual manufacturera del DANE pueden dar una idea equivocada del movimiento en la producción real, si el número de días hábiles mensuales cambia de un año a otro y no se efectúan los ajustes apropiados.

Para algunos meses, la diferencia entre la cifra de crecimiento industrial reportada por el DANE y la cifra ajustada por días hábiles, puede llegar a los 12 puntos porcentuales en valor absoluto.

Debe subrayarse que el problema del ajuste por días hábiles no se limita al efecto de Semana Santa en los meses 
de marzo y abril. Otros meses del año tienen importantes variaciones de un año a otro en su número de días hábiles.

Este trabajo presenta una metodología que puede ser utilizada por los investigadores para ajustar las cifras de producción industrial; es aplicable igualmente a otras variables económicas cuya magnitud dependa del número de días hábiles mensuales, trimestrales o anuales.

\section{Referencias}

Banco de la República. (2005, junio). Informe sobre la Inflación. Bogotá: Banrep.

Colombia, Congreso de la República. (1983, 22 de dic.). Ley 51 o Ley Emiliani. Por la cual se traslada el descanso remunerado de algunos días festivos. En Diario Oficial No. 36.428, (1983, 30 de dic.).

Cuando en el Mundo. (2008-2014). Calendario. Recuperado de http://www.cuandoenel mundo.com/calendario/

Eurostat. (2006). Methodology of Short-term Business Statistics. Interpretation and Guidelines. Luxemburgo: Office for Official Publications of the European Communities.

Federal Reserve. (2003, abril). Industrial Production and Capacity Utilization: The 2002 Historical and Annual Revision. Federal Reserve Bulletin, 89.

Landiray, D. (2006, mayo). Calendar Effect and Seasonal Adjustment, Eurostat Workshop (1012). INSEE.

Martínez, C. (2004). Pronósticos de la producción industrial. Documento 273. Archivos de Macroeconomía. Bogotá: Departamento Nacional de Planeación, Dirección de Estudios Económicos.

Montenegro, A. (2010). Cifras de producción industrial ajustadas por días hábiles. Documento de trabajo. Departamento de Economía, Universidad Javeriana. Universitas Económica, (1).

Montenegro, A. (2011). Análisis de series de tiempo, Bogotá: Editorial Universidad Javeriana.

Soukup, R. \& Findley, D. (2000, oct.). Modeling and Model Selection for Moving Holidays. US Census Bureau, Seasonal Adjustment Papers.

Statistics Canada. (2014, 31 de marzo). Gross Domestic Product by Industry, National Monthly. Data Release. Retrieved from http://www23.statcan.gc.ca/imdb/p2SV.pl? Function $=$ getSurvey $\&$ SDDS $=1301$ 
Series de días hábiles en Colombia: una aplicación al ajuste del crecimiento industrial Álvaro Montenegro García

ANEXO

Días hábiles mensuales (con sábados) y cambio porcentual con el mismo mes del año anterior

\begin{tabular}{|c|c|c|c|c|c|c|c|c|}
\hline Fecha & $\begin{array}{c}\text { Días } \\
\text { Hábiles }\end{array}$ & $\begin{array}{c}\text { Cambio } \\
\text { Porcentual }\end{array}$ & Fecha & $\begin{array}{c}\text { Días } \\
\text { Hábiles }\end{array}$ & $\begin{array}{c}\text { Cambio } \\
\text { Porcentual }\end{array}$ & Fecha & $\begin{array}{c}\text { Días } \\
\text { Hábiles }\end{array}$ & $\begin{array}{c}\text { Cambio } \\
\text { Porcentual }\end{array}$ \\
\hline 1980M01 & 26 & $\mathrm{Na}$ & $1983 \mathrm{M} 01$ & 24 & 0.0000 & $1986 \mathrm{M} 01$ & 25 & 0.0000 \\
\hline 1980M02 & 25 & $\mathrm{Na}$ & 1983M02 & 24 & 0.0000 & 1986M02 & 24 & 0.0000 \\
\hline 1980M03 & 25 & $\mathrm{Na}$ & $1983 \mathrm{M} 03$ & 25 & -3.8462 & $1986 \mathrm{M} 03$ & 23 & -8.0000 \\
\hline 1980M04 & 24 & $\mathrm{Na}$ & $1983 \mathrm{M} 04$ & 25 & 4.1667 & 1986M04 & 26 & 8.3333 \\
\hline 1980M05 & 25 & $\mathrm{Na}$ & 1983M05 & 25 & 4.1667 & 1986M05 & 25 & 0.0000 \\
\hline 1980M06 & 23 & $\mathrm{Na}$ & $1983 \mathrm{M} 06$ & 23 & 0.0000 & 1986M06 & 22 & -4.3478 \\
\hline 1980M07 & 27 & $\mathrm{Na}$ & $1983 \mathrm{M} 07$ & 25 & -3.8462 & 1986M07 & 27 & 8.0000 \\
\hline 1980M08 & 24 & $\mathrm{Na}$ & 1983M08 & 26 & 4.0000 & 1986M08 & 24 & -4.0000 \\
\hline 1980M09 & 26 & $\mathrm{Na}$ & 1983M09 & 26 & 0.0000 & 1986M09 & 26 & 4.0000 \\
\hline 1980M10 & 27 & $\mathrm{Na}$ & $1983 \mathrm{M} 10$ & 25 & 0.0000 & $1986 \mathrm{M} 10$ & 26 & 0.0000 \\
\hline 1980M11 & 23 & $\mathrm{Na}$ & $1983 \mathrm{M} 11$ & 24 & 0.0000 & $1986 \mathrm{M} 11$ & 23 & -4.1667 \\
\hline $1980 \mathrm{M} 12$ & 25 & $\mathrm{Na}$ & $1983 \mathrm{M} 12$ & 26 & 4.0000 & $1986 \mathrm{M} 12$ & 25 & 0.0000 \\
\hline 1981M01 & 25 & -3.8462 & 1984M01 & 25 & 4.1667 & $1987 \mathrm{M} 01$ & 25 & 0.0000 \\
\hline 1981M02 & 24 & -4.0000 & 1984M02 & 25 & 4.1667 & $1987 \mathrm{M} 02$ & 24 & 0.0000 \\
\hline $1981 \mathrm{M} 03$ & 25 & 0.0000 & $1984 \mathrm{M} 03$ & 26 & 4.0000 & $1987 \mathrm{M} 03$ & 25 & 8.6957 \\
\hline $1981 \mathrm{M} 04$ & 24 & 0.0000 & 1984M04 & 23 & -8.0000 & $1987 \mathrm{M} 04$ & 24 & -7.6923 \\
\hline 1981M05 & 24 & -4.0000 & 1984M05 & 26 & 4.0000 & $1987 \mathrm{M} 05$ & 25 & 0.0000 \\
\hline 1981M06 & 23 & 0.0000 & 1984M06 & 24 & 4.3478 & 1987M06 & 23 & 4.5455 \\
\hline 1981M07 & 26 & -3.7037 & 1984M07 & 24 & -4.0000 & 1987M07 & 26 & -3.7037 \\
\hline 1981M08 & 24 & 0.0000 & 1984M08 & 25 & -3.8462 & $1987 \mathrm{M} 08$ & 24 & 0.0000 \\
\hline 1981M09 & 26 & 0.0000 & 1984M09 & 25 & -3.8462 & 1987M09 & 26 & 0.0000 \\
\hline $1981 \mathrm{M} 10$ & 26 & -3.7037 & $1984 \mathrm{M} 10$ & 26 & 4.0000 & $1987 \mathrm{M} 10$ & 26 & 0.0000 \\
\hline $1981 \mathrm{M} 11$ & 24 & 4.3478 & $1984 \mathrm{M} 11$ & 24 & 0.0000 & $1987 \mathrm{M} 11$ & 23 & 0.0000 \\
\hline $1981 \mathrm{M} 12$ & 25 & 0.0000 & $1984 \mathrm{M} 12$ & 24 & -7.6923 & $1987 \mathrm{M} 12$ & 25 & 0.0000 \\
\hline 1982M01 & 24 & -4.0000 & $1985 \mathrm{M} 01$ & 25 & 0.0000 & $1988 \mathrm{M} 01$ & 24 & -4.0000 \\
\hline 1982M02 & 24 & 0.0000 & $1985 \mathrm{M} 02$ & 24 & -4.0000 & $1988 \mathrm{M} 02$ & 25 & 4.1667 \\
\hline 1982M03 & 26 & 4.0000 & $1985 \mathrm{M} 03$ & 25 & -3.8462 & $1988 \mathrm{M} 03$ & 25 & 0.0000 \\
\hline 1982M04 & 24 & 0.0000 & $1985 \mathrm{M} 04$ & 24 & 4.3478 & 1988M04 & 25 & 4.1667 \\
\hline 1982M05 & 24 & 0.0000 & $1985 \mathrm{M} 05$ & 25 & -3.8462 & $1988 \mathrm{M} 05$ & 25 & 0.0000 \\
\hline 1982M06 & 23 & 0.0000 & $1985 \mathrm{M} 06$ & 23 & -4.1667 & 1988M06 & 24 & 4.3478 \\
\hline 1982M07 & 26 & 0.0000 & $1985 \mathrm{M} 07$ & 25 & 4.1667 & 1988M07 & 24 & -7.6923 \\
\hline 1982M08 & 25 & 4.1667 & $1985 \mathrm{M} 08$ & 25 & 0.0000 & $1988 \mathrm{M} 08$ & 26 & 8.3333 \\
\hline 1982M09 & 26 & 0.0000 & $1985 \mathrm{M} 09$ & 25 & 0.0000 & 1988M09 & 26 & 0.0000 \\
\hline $1982 \mathrm{M} 10$ & 25 & -3.8462 & $1985 \mathrm{M} 10$ & 26 & 0.0000 & $1988 \mathrm{M} 10$ & 25 & -3.8462 \\
\hline 1982M11 & 24 & 0.0000 & $1985 \mathrm{M} 11$ & 24 & 0.0000 & $1988 \mathrm{M} 11$ & 24 & 4.3478 \\
\hline $1982 \mathrm{M} 12$ & 25 & 0.0000 & $1985 \mathrm{M} 12$ & 25 & 4.1667 & $1988 \mathrm{M} 12$ & 26 & 4.0000 \\
\hline
\end{tabular}




\begin{tabular}{|c|c|c|c|c|c|c|c|c|}
\hline Fecha & $\begin{array}{c}\text { Días } \\
\text { Hábiles }\end{array}$ & $\begin{array}{c}\text { Cambio } \\
\text { Porcentual }\end{array}$ & Fecha & $\begin{array}{c}\text { Días } \\
\text { Hábiles }\end{array}$ & $\begin{array}{c}\text { Cambio } \\
\text { Porcentual }\end{array}$ & Fecha & $\begin{array}{c}\text { Días } \\
\text { Hábiles }\end{array}$ & $\begin{array}{c}\text { Cambio } \\
\text { Porcentual }\end{array}$ \\
\hline 1989M01 & 25 & 4.1667 & 1992M06 & 23 & 0.0000 & $1995 \mathrm{M} 11$ & 24 & 0.0000 \\
\hline 1989M02 & 24 & -4.0000 & 1992M07 & 26 & 4.0000 & $1995 \mathrm{M} 12$ & 24 & -7.6923 \\
\hline 1989M03 & 24 & -4.0000 & 1992M08 & 24 & -4.0000 & 1996M01 & 25 & 0.0000 \\
\hline 1989M04 & 25 & 0.0000 & 1992M09 & 26 & 4.0000 & 1996M02 & 25 & 4.1667 \\
\hline 1989M05 & 24 & -4.0000 & $1992 \mathrm{M} 10$ & 26 & 0.0000 & 1996M03 & 25 & -3.8462 \\
\hline 1989M06 & 25 & 4.1667 & 1992M11 & 23 & -4.1667 & 1996M04 & 24 & 4.3478 \\
\hline 1989M07 & 24 & 0.0000 & $1992 \mathrm{M} 12$ & 25 & 0.0000 & 1996M05 & 25 & 0.0000 \\
\hline 1989M08 & 25 & -3.8462 & $1993 \mathrm{M} 01$ & 24 & -4.0000 & 1996M06 & 23 & -4.1667 \\
\hline 1989M09 & 26 & 0.0000 & 1993M02 & 24 & -4.0000 & 1996M07 & 25 & 4.1667 \\
\hline 1989M10 & 25 & 0.0000 & $1993 \mathrm{M} 03$ & 26 & 4.0000 & 1996M08 & 25 & 0.0000 \\
\hline 1989M11 & 24 & 0.0000 & $1993 \mathrm{M} 04$ & 24 & 0.0000 & 1996M09 & 25 & -3.8462 \\
\hline 1989M12 & 24 & -7.6923 & $1993 \mathrm{M} 05$ & 24 & -4.0000 & 1996M10 & 26 & 4.0000 \\
\hline 1990M01 & 25 & 0.0000 & 1993M06 & 24 & 4.3478 & 1996M11 & 24 & 0.0000 \\
\hline 1990M02 & 24 & 0.0000 & $1993 \mathrm{M} 07$ & 25 & -3.8462 & 1996M12 & 25 & 4.1667 \\
\hline 1990M03 & 26 & 8.3333 & $1993 \mathrm{M} 08$ & 24 & 0.0000 & 1997M01 & 25 & 0.0000 \\
\hline 1990M04 & 23 & -8.0000 & $1993 \mathrm{M} 09$ & 26 & 0.0000 & 1997M02 & 24 & -4.0000 \\
\hline 1990M05 & 25 & 4.1667 & $1993 \mathrm{M} 10$ & 25 & -3.8462 & 1997M03 & 23 & -8.0000 \\
\hline 1990M06 & 24 & -4.0000 & $1993 \mathrm{M} 11$ & 24 & 4.3478 & 1997M04 & 26 & 8.3333 \\
\hline 1990M07 & 24 & 0.0000 & $1993 \mathrm{M} 12$ & 25 & 0.0000 & 1997M05 & 25 & 0.0000 \\
\hline 1990M08 & 25 & 0.0000 & 1994M01 & 24 & 0.0000 & 1997M06 & 22 & -4.3478 \\
\hline 1990M09 & 25 & -3.8462 & 1994M02 & 24 & 0.0000 & 1997M07 & 27 & 8.0000 \\
\hline 1990M10 & 26 & 4.0000 & 1994M03 & 25 & -3.8462 & 1997M08 & 24 & -4.0000 \\
\hline 1990M11 & 24 & 0.0000 & 1994M04 & 25 & 4.1667 & 1997M09 & 26 & 4.0000 \\
\hline 1990M12 & 24 & 0.0000 & 1994M05 & 25 & 4.1667 & $1997 \mathrm{M} 10$ & 26 & 0.0000 \\
\hline 1991M01 & 25 & 0.0000 & 1994M06 & 24 & 0.0000 & 1997M11 & 23 & -4.1667 \\
\hline 1991M02 & 24 & 0.0000 & 1994M07 & 24 & -4.0000 & 1997M12 & 25 & 0.0000 \\
\hline 1991M03 & 23 & -11.5385 & 1994M08 & 26 & 8.3333 & 1998M01 & 25 & 0.0000 \\
\hline 1991M04 & 26 & 13.0435 & 1994M09 & 26 & 0.0000 & 1998M02 & 24 & 0.0000 \\
\hline 1991M05 & 25 & 0.0000 & $1994 \mathrm{M} 10$ & 25 & 0.0000 & 1998M03 & 25 & 8.6957 \\
\hline 1991M06 & 23 & -4.1667 & $1994 \mathrm{M} 11$ & 24 & 0.0000 & 1998M04 & 24 & -7.6923 \\
\hline 1991M07 & 25 & 4.1667 & $1994 \mathrm{M} 12$ & 26 & 4.0000 & 1998M05 & 24 & -4.0000 \\
\hline 1991M08 & 25 & 0.0000 & $1995 \mathrm{M} 01$ & 25 & 4.1667 & 1998M06 & 23 & 4.5455 \\
\hline 1991M09 & 25 & 0.0000 & $1995 \mathrm{M} 02$ & 24 & 0.0000 & 1998M07 & 26 & -3.7037 \\
\hline 1991M10 & 26 & 0.0000 & $1995 \mathrm{M} 03$ & 26 & 4.0000 & 1998M08 & 24 & 0.0000 \\
\hline 1991M11 & 24 & 0.0000 & $1995 \mathrm{M} 04$ & 23 & -8.0000 & 1998M09 & 26 & 0.0000 \\
\hline 1991M12 & 25 & 4.1667 & $1995 \mathrm{M} 05$ & 25 & 0.0000 & 1998M10 & 26 & 0.0000 \\
\hline 1992M01 & 25 & 0.0000 & $1995 \mathrm{M} 06$ & 24 & 0.0000 & 1998M11 & 23 & 0.0000 \\
\hline 1992M02 & 25 & 4.1667 & $1995 \mathrm{M} 07$ & 24 & 0.0000 & 1998M12 & 25 & 0.0000 \\
\hline 1992M03 & 25 & 8.6957 & 1995M08 & 25 & -3.8462 & 1999M01 & 24 & -4.0000 \\
\hline 1992M04 & 24 & -7.6923 & $1995 \mathrm{M} 09$ & 26 & 0.0000 & 1999M02 & 24 & 0.0000 \\
\hline 1992M05 & 25 & 0.0000 & $1995 \mathrm{M} 10$ & 25 & 0.0000 & 1999M03 & 26 & 4.0000 \\
\hline
\end{tabular}




\begin{tabular}{|c|c|c|c|c|c|c|c|c|}
\hline Fecha & $\begin{array}{c}\text { Días } \\
\text { Hábiles }\end{array}$ & $\begin{array}{c}\text { Cambio } \\
\text { Porcentual }\end{array}$ & Fecha & $\begin{array}{c}\text { Días } \\
\text { Hábiles }\end{array}$ & $\begin{array}{c}\text { Cambio } \\
\text { Porcentual }\end{array}$ & Fecha & $\begin{array}{c}\text { Días } \\
\text { Hábiles }\end{array}$ & $\begin{array}{c}\text { Cambio } \\
\text { Porcentual }\end{array}$ \\
\hline 1999M04 & 24 & 0.0000 & $2002 \mathrm{M} 09$ & 25 & 0.0000 & 2006M02 & 24 & 0.0000 \\
\hline 1999M05 & 24 & 0.0000 & $2002 \mathrm{M} 10$ & 26 & 0.0000 & 2006M03 & 26 & 8.3333 \\
\hline 1999M06 & 24 & 4.3478 & $2002 \mathrm{M} 11$ & 24 & 0.0000 & 2006M04 & 23 & -11.5385 \\
\hline 1999M07 & 25 & -3.8462 & $2002 \mathrm{M} 12$ & 25 & 4.1667 & 2006M05 & 25 & 4.1667 \\
\hline 1999M08 & 24 & 0.0000 & $2003 \mathrm{M} 01$ & 25 & 0.0000 & 2006M06 & 24 & -4.0000 \\
\hline 1999M09 & 26 & 0.0000 & $2003 \mathrm{M} 02$ & 24 & 0.0000 & 2006M07 & 24 & 0.0000 \\
\hline 1999M10 & 25 & -3.8462 & $2003 \mathrm{M} 03$ & 25 & 8.6957 & 2006M08 & 25 & -3.8462 \\
\hline 1999M11 & 24 & 4.3478 & $2003 \mathrm{M} 04$ & 24 & -7.6923 & 2006M09 & 26 & 0.0000 \\
\hline 1999M12 & 25 & 0.0000 & $2003 \mathrm{M} 05$ & 26 & 4.0000 & $2006 \mathrm{M} 10$ & 25 & 0.0000 \\
\hline 2000M01 & 24 & 0.0000 & $2003 \mathrm{M} 06$ & 22 & -4.3478 & $2006 \mathrm{M} 11$ & 24 & 0.0000 \\
\hline 2000M02 & 25 & 4.1667 & $2003 \mathrm{M} 07$ & 27 & 8.0000 & $2006 \mathrm{M} 12$ & 24 & -7.6923 \\
\hline 2000M03 & 26 & 0.0000 & $2003 \mathrm{M} 08$ & 24 & -4.0000 & 2007M01 & 25 & 0.0000 \\
\hline 2000M04 & 23 & -4.1667 & $2003 \mathrm{M} 09$ & 26 & 4.0000 & $2007 \mathrm{M} 02$ & 24 & 0.0000 \\
\hline 2000M05 & 26 & 8.3333 & $2003 \mathrm{M} 10$ & 26 & 0.0000 & $2007 \mathrm{M} 03$ & 26 & 0.0000 \\
\hline 2000M06 & 24 & 0.0000 & $2003 \mathrm{M} 11$ & 23 & -4.1667 & 2007M04 & 23 & 0.0000 \\
\hline 2000M07 & 24 & -4.0000 & $2003 \mathrm{M} 12$ & 25 & 0.0000 & 2007M05 & 25 & 0.0000 \\
\hline 2000M08 & 25 & 4.1667 & $2004 \mathrm{M} 01$ & 25 & 0.0000 & 2007M06 & 24 & 0.0000 \\
\hline 2000M09 & 26 & 0.0000 & 2004M02 & 24 & 0.0000 & 2007M07 & 24 & 0.0000 \\
\hline 2000M10 & 25 & 0.0000 & 2004M03 & 26 & 4.0000 & 2007M08 & 25 & 0.0000 \\
\hline 2000M11 & 24 & 0.0000 & $2004 \mathrm{M} 04$ & 24 & 0.0000 & 2007M09 & 25 & -3.8462 \\
\hline $2000 \mathrm{M} 12$ & 24 & -4.0000 & $2004 \mathrm{M} 05$ & 24 & -7.6923 & $2007 \mathrm{M} 10$ & 26 & 4.0000 \\
\hline 2001M01 & 25 & 4.1667 & 2004M06 & 24 & 9.0909 & $2007 \mathrm{M} 11$ & 24 & 0.0000 \\
\hline 2001M02 & 24 & -4.0000 & 2004M07 & 25 & -7.4074 & $2007 \mathrm{M} 12$ & 24 & 0.0000 \\
\hline 2001M03 & 26 & 0.0000 & 2004M08 & 24 & 0.0000 & 2008M01 & 25 & 0.0000 \\
\hline 2001M04 & 23 & 0.0000 & 2004M09 & 26 & 0.0000 & 2008M02 & 25 & 4.1667 \\
\hline 2001M05 & 25 & -3.8462 & $2004 \mathrm{M} 10$ & 25 & -3.8462 & $2008 \mathrm{M} 03$ & 23 & -11.5385 \\
\hline 2001M06 & 24 & 0.0000 & $2004 \mathrm{M} 11$ & 24 & 4.3478 & 2008M04 & 26 & 13.0435 \\
\hline 2001M07 & 24 & 0.0000 & $2004 \mathrm{M} 12$ & 25 & 0.0000 & 2008M05 & 24 & -4.0000 \\
\hline 2001M08 & 25 & 0.0000 & $2005 \mathrm{M} 01$ & 24 & -4.0000 & 2008M06 & 23 & -4.1667 \\
\hline 2001M09 & 25 & -3.8462 & $2005 \mathrm{M} 02$ & 24 & 0.0000 & 2008M07 & 27 & 12.5000 \\
\hline $2001 \mathrm{M} 10$ & 26 & 4.0000 & $2005 \mathrm{M} 03$ & 24 & -7.6923 & 2008M08 & 24 & -4.0000 \\
\hline 2001M11 & 24 & 0.0000 & $2005 \mathrm{M} 04$ & 26 & 8.3333 & 2008M09 & 26 & 4.0000 \\
\hline $2001 \mathrm{M} 12$ & 24 & 0.0000 & $2005 \mathrm{M} 05$ & 24 & 0.0000 & $2008 \mathrm{M} 10$ & 26 & 0.0000 \\
\hline $2002 \mathrm{M} 01$ & 25 & 0.0000 & $2005 \mathrm{M} 06$ & 25 & 4.1667 & 2008M11 & 23 & -4.1667 \\
\hline 2002M02 & 24 & 0.0000 & $2005 \mathrm{M} 07$ & 24 & -4.0000 & 2008M12 & 25 & 4.1667 \\
\hline 2002M03 & 23 & -11.5385 & $2005 \mathrm{M} 08$ & 26 & 8.3333 & 2009M01 & 25 & 0.0000 \\
\hline 2002M04 & 26 & 13.0435 & $2005 \mathrm{M} 09$ & 26 & 0.0000 & 2009M02 & 24 & -4.0000 \\
\hline 2002M05 & 25 & 0.0000 & $2005 \mathrm{M} 10$ & 25 & 0.0000 & 2009M03 & 25 & 8.6957 \\
\hline 2002M06 & 23 & -4.1667 & $2005 \mathrm{M} 11$ & 24 & 0.0000 & 2009M04 & 24 & -7.6923 \\
\hline 2002M07 & 25 & 4.1667 & $2005 \mathrm{M} 12$ & 26 & 4.0000 & 2009M05 & 24 & 0.0000 \\
\hline 2002M08 & 25 & 0.0000 & $2006 \mathrm{M} 01$ & 25 & 4.1667 & 2009M06 & 23 & 0.0000 \\
\hline
\end{tabular}




\begin{tabular}{|c|c|c|c|c|c|c|c|c|}
\hline Fecha & $\begin{array}{c}\text { Días } \\
\text { Hábiles }\end{array}$ & $\begin{array}{c}\text { Cambio } \\
\text { Porcentual }\end{array}$ & Fecha & $\begin{array}{c}\text { Días } \\
\text { Hábiles }\end{array}$ & $\begin{array}{c}\text { Cambio } \\
\text { Porcentual }\end{array}$ & Fecha & $\begin{array}{c}\text { Días } \\
\text { Hábiles }\end{array}$ & $\begin{array}{c}\text { Cambio } \\
\text { Porcentual }\end{array}$ \\
\hline 2009M07 & 26 & -3.7037 & $2012 \mathrm{M} 12$ & 24 & -7.6923 & 2016M05 & 24 & 0.0000 \\
\hline 2009M08 & 24 & 0.0000 & $2013 \mathrm{M} 01$ & 25 & 0.0000 & 2016M06 & 25 & 8.6957 \\
\hline 2009M09 & 26 & 0.0000 & $2013 \mathrm{M} 02$ & 24 & -4.0000 & 2016M07 & 24 & -7.6923 \\
\hline 2009M10 & 26 & 0.0000 & $2013 \mathrm{M} 03$ & 23 & -11.5385 & 2016M08 & 26 & 8.3333 \\
\hline 2009M11 & 23 & 0.0000 & $2013 \mathrm{M} 04$ & 26 & 13.0435 & 2016M09 & 26 & 0.0000 \\
\hline 2009M12 & 25 & 0.0000 & $2013 \mathrm{M} 05$ & 25 & 0.0000 & $2016 \mathrm{M} 10$ & 25 & -3.8462 \\
\hline 2010M01 & 24 & -4.0000 & $2013 \mathrm{M} 06$ & 23 & -4.1667 & 2016M11 & 24 & 4.3478 \\
\hline 2010M02 & 24 & 0.0000 & $2013 \mathrm{M} 07$ & 25 & 4.1667 & $2016 \mathrm{M} 12$ & 26 & 4.0000 \\
\hline 2010M03 & 26 & 4.0000 & $2013 \mathrm{M} 08$ & 25 & 0.0000 & 2017M01 & 25 & 4.1667 \\
\hline 2010M04 & 24 & 0.0000 & 2013M09 & 25 & 0.0000 & 2017M02 & 24 & -4.0000 \\
\hline 2010M05 & 24 & 0.0000 & $2013 \mathrm{M} 10$ & 26 & 0.0000 & $2017 \mathrm{M} 03$ & 26 & 8.3333 \\
\hline 2010M06 & 24 & 4.3478 & $2013 \mathrm{M} 11$ & 24 & 0.0000 & 2017M04 & 23 & -11.5385 \\
\hline 2010M07 & 25 & -3.8462 & $2013 \mathrm{M} 12$ & 25 & 4.1667 & 2017M05 & 25 & 4.1667 \\
\hline 2010M08 & 24 & 0.0000 & $2014 \mathrm{M} 01$ & 25 & 0.0000 & 2017M06 & 24 & -4.0000 \\
\hline 2010M09 & 26 & 0.0000 & $2014 \mathrm{M} 02$ & 24 & 0.0000 & 2017M07 & 24 & 0.0000 \\
\hline 2010M10 & 25 & -3.8462 & $2014 \mathrm{M} 03$ & 25 & 8.6957 & 2017M08 & 25 & -3.8462 \\
\hline 2010M11 & 24 & 4.3478 & $2014 \mathrm{M} 04$ & 24 & -7.6923 & 2017M09 & 26 & 0.0000 \\
\hline 2010M12 & 25 & 0.0000 & $2014 \mathrm{M} 05$ & 26 & 4.0000 & $2017 \mathrm{M} 10$ & 25 & 0.0000 \\
\hline 2011M01 & 24 & 0.0000 & $2014 \mathrm{M} 06$ & 22 & -4.3478 & $2017 \mathrm{M} 11$ & 24 & 0.0000 \\
\hline 2011M02 & 24 & 0.0000 & $2014 \mathrm{M} 07$ & 27 & 8.0000 & $2017 \mathrm{M} 12$ & 24 & -7.6923 \\
\hline 2011M03 & 26 & 0.0000 & 2014M08 & 24 & -4.0000 & 2018M01 & 25 & 0.0000 \\
\hline 2011M04 & 24 & 0.0000 & 2014M09 & 26 & 4.0000 & 2018M02 & 24 & 0.0000 \\
\hline 2011M05 & 26 & 8.3333 & $2014 \mathrm{M} 10$ & 26 & 0.0000 & 2018M03 & 24 & -7.6923 \\
\hline 2011M06 & 24 & 0.0000 & $2014 \mathrm{M} 11$ & 23 & -4.1667 & 2018M04 & 25 & 8.6957 \\
\hline 2011M07 & 24 & -4.0000 & $2014 \mathrm{M} 12$ & 25 & 0.0000 & 2018M05 & 25 & 0.0000 \\
\hline 2011M08 & 26 & 8.3333 & $2015 \mathrm{M} 01$ & 25 & 0.0000 & 2018M06 & 24 & 0.0000 \\
\hline 2011M09 & 26 & 0.0000 & $2015 \mathrm{M} 02$ & 24 & 0.0000 & 2018M07 & 24 & 0.0000 \\
\hline $2011 \mathrm{M} 10$ & 25 & 0.0000 & $2015 \mathrm{M} 03$ & 25 & 0.0000 & 2018M08 & 25 & 0.0000 \\
\hline 2011M11 & 24 & 0.0000 & $2015 \mathrm{M} 04$ & 24 & 0.0000 & 2018M09 & 25 & -3.8462 \\
\hline $2011 \mathrm{M} 12$ & 26 & 4.0000 & $2015 \mathrm{M} 05$ & 24 & -7.6923 & $2018 \mathrm{M} 10$ & 26 & 4.0000 \\
\hline 2012M01 & 25 & 4.1667 & $2015 \mathrm{M} 06$ & 23 & 4.5455 & 2018M11 & 24 & 0.0000 \\
\hline 2012M02 & 25 & 4.1667 & $2015 \mathrm{M} 07$ & 26 & -3.7037 & 2018M12 & 24 & 0.0000 \\
\hline 2012M03 & 26 & 0.0000 & $2015 \mathrm{M} 08$ & 24 & 0.0000 & 2019M01 & 25 & 0.0000 \\
\hline 2012M04 & 23 & -4.1667 & $2015 \mathrm{M} 09$ & 26 & 0.0000 & 2019M02 & 24 & 0.0000 \\
\hline 2012M05 & 25 & -3.8462 & $2015 \mathrm{M} 10$ & 26 & 0.0000 & 2019M03 & 25 & 4.1667 \\
\hline 2012M06 & 24 & 0.0000 & $2015 \mathrm{M} 11$ & 23 & 0.0000 & 2019M04 & 24 & -4.0000 \\
\hline 2012M07 & 24 & 0.0000 & $2015 \mathrm{M} 12$ & 25 & 0.0000 & 2019M05 & 26 & 4.0000 \\
\hline 2012M08 & 25 & -3.8462 & $2016 \mathrm{M} 01$ & 24 & -4.0000 & 2019M06 & 23 & -4.1667 \\
\hline 2012M09 & 25 & -3.8462 & $2016 \mathrm{M} 02$ & 25 & 4.1667 & 2019M07 & 25 & 4.1667 \\
\hline $2012 \mathrm{M} 10$ & 26 & 4.0000 & $2016 \mathrm{M} 03$ & 24 & -4.0000 & 2019M08 & 25 & 0.0000 \\
\hline 2012M11 & 24 & 0.0000 & 2016M04 & 26 & 8.3333 & 2019M09 & 25 & 0.0000 \\
\hline
\end{tabular}


Series de días hábiles en Colombia: una aplicación al ajuste del crecimiento industrial

Álvaro Montenegro García

$\begin{array}{ccccccccc}\text { Fecha } & \begin{array}{c}\text { Días } \\ \text { Hábiles }\end{array} & \begin{array}{c}\text { Cambio } \\ \text { Porcentual }\end{array} & \text { Fecha } & \begin{array}{c}\text { Días } \\ \text { Hábiles Porcentual }\end{array} & \begin{array}{c}\text { Cambio } \\ \text { Fecha }\end{array} & \begin{array}{c}\text { Días } \\ \text { Hábiles }\end{array} & \begin{array}{c}\text { Cambio } \\ \text { Porcentual }\end{array} \\ 2019 \mathrm{M} 10 & 26 & 0.0000 & 2020 \mathrm{M} 07 & 26 & 4.0000 & 2021 \mathrm{M} 04 & 24 & 0.0000 \\ 2019 \mathrm{M} 11 & 24 & 0.0000 & 2020 \mathrm{M} 08 & 24 & -4.0000 & 2021 \mathrm{M} 05 & 24 & 0.0000 \\ 2019 \mathrm{M} 12 & 25 & 4.1667 & 2020 \mathrm{M} 09 & 26 & 4.0000 & 2021 \mathrm{M} 06 & 24 & 4.3478 \\ 2020 \mathrm{M} 01 & 25 & 0.0000 & 2020 \mathrm{M} 10 & 26 & 0.0000 & 2021 \mathrm{M} 07 & 25 & -3.8462 \\ 2020 \mathrm{M} 02 & 25 & 4.1667 & 2020 \mathrm{M} 11 & 23 & -4.1667 & 2021 \mathrm{M} 08 & 24 & 0.0000 \\ 2020 \mathrm{M} 03 & 25 & 0.0000 & 2020 \mathrm{M} 12 & 25 & 0.0000 & 2021 \mathrm{M} 09 & 26 & 0.0000 \\ 2020 \mathrm{M} 04 & 24 & 0.0000 & 2021 \mathrm{M} 01 & 24 & -4.0000 & 2021 \mathrm{M} 10 & 25 & -3.8462 \\ 2020 \mathrm{M} 05 & 24 & -7.6923 & 2021 \mathrm{M} 02 & 24 & -4.0000 & 2021 \mathrm{M} 11 & 24 & 4.3478 \\ 2020 \mathrm{M} 06 & 23 & 0.0000 & 2021 \mathrm{M} 03 & 26 & 4.0000 & 2021 \mathrm{M} 12 & 25 & 0.0000\end{array}$

